\section{THE STRUCTURAL ELUCIDATION OF AMINOGLYCOSIDE ANTIBIOTICS, SANNAMYCINS A AND B}

Sir :

Sannamycins A and B are new aminoglycoside antibiotics produced by Streptomyces sannanensis strain KC-7038 sp. nov.

The production, isolation, physico-chemical and biological properties of sannamycins have been described previously ${ }^{1)}$. In this communication, the structural elucidations of these antibiotics are described.

Sannamycins A (1) and B (2) are obtained as a colorless powder and show no definite melting points. The former (1) shows $[\alpha]_{\mathrm{D}}^{25}+120.5^{\circ}$ $\left(c 1, \mathrm{H}_{2} \mathrm{O}\right.$ ) and the latter $(2)$ shows $[\alpha]_{1}^{25}+78^{\circ}$ (c $0.5, \mathrm{H}_{2} \mathrm{O}$ ). The formulae $\mathrm{C}_{17} \mathrm{H}_{35} \mathrm{~N}_{5} \mathrm{O}_{5}$ and $\mathrm{C}_{15} \mathrm{H}_{32} \mathrm{~N}_{4} \mathrm{O}_{4}$ were established for $\mathbf{1}$ and 2 by mass spectrometry, ${ }^{13} \mathrm{C}$ NMR spectra and elemental analysis.

The mass spectrometry of 1 or 2 showed a highly intensity peak at $m / e$ 143. The ${ }^{1} \mathrm{H}$ NMR spectra of $\mathbf{1}$ and $\mathbf{2}$ in $\mathrm{D}_{2} \mathrm{O}$ showed three methyl groups and one anomeric proton signal, as shown in Table 1.

The most significant difference between $\mathbf{1}$ and $\mathbf{2}$ is the marked deshielding of one of the $\mathrm{N}$-methyl resonances in $\mathbf{1}$. On the basis of their physical properties and their empirical formulae, which coincide with those of sporaricins $\mathrm{A}$ and $\mathrm{B}^{21}$ respectively, we assumed that sannamycins A and B were similar to the sporaricins $\mathrm{A}$ and $\mathrm{B}$ and fortimicins $\mathrm{A}$ and $\mathrm{B}^{3)}$. $\mathrm{Al}-$ kaline hydrolysis of 1 (145 mg, $0.37 \mathrm{mmol}$ ) with $1 \mathrm{~N} \mathrm{Ba}(\mathrm{OH})_{2}$ $(20 \mathrm{ml})$ under reflux for 4 hours afforded $2(60 \mathrm{mg})$ and glycine. These facts proved that $\mathbf{1}$ and $\mathbf{2}$ were pseudodisaccharides and $\mathbf{1}$ was the mono glycylated analog of 2.

Acetylation of 2 (664 mg, 2 $\mathrm{mmol}$ ) with acetic anhydride in methanol by standing overnight at room temperature gave the
tetra-N-acetyl derivative (3) quantitatively, $\mathrm{m} / \mathrm{e}$ $500 \mathrm{M}^{+},[\alpha]_{\mathrm{D}}^{23}+189^{\circ}\left(c 1, \mathrm{H}_{2} \mathrm{O}\right)$.

Methanolysis of $3(500 \mathrm{mg}, 1 \mathrm{mmol})$ with $6 \mathrm{~N}$ hydrogen chloride in anhydrous methanol (20 $\mathrm{ml}, 80^{\circ} \mathrm{C}, 8$ hours in a sealed tube) followed by re-N-acetylation afforded three spots except for unreacted 3 on silica-gel thin-layer chromatography developed with chloroform-methanol $(8: 1)$. The reaction mixture was evaporated under reduced pressure and chromatographed on a column of silica gel. The column was developed with chloroform - acetone $(1: 2)$ to give a methyl $\mathrm{N}$-acetyl- $\alpha$-glycoside $\left(4,120 \mathrm{mg},[\alpha]^{24}+142^{\circ}\right.$ (c $1, \mathrm{H}_{2} \mathrm{O}$ ), m/e $258 \mathrm{M}^{+}$, Anal. $\mathrm{C}_{12} \mathrm{H}_{22} \mathrm{~N}_{2} \mathrm{O}_{4}$ ) and the anomer $\left(5,52 \mathrm{mg},[\alpha]_{i}^{24} 0^{\circ}\left(c 1, \mathrm{H}_{2} \mathrm{O}\right)\right.$, $\left.m / e 258 \mathrm{M}^{+}\right)$as colorless syrups and a colorless solid of an $\mathrm{N}$-acetylaminocyclitol $(6,201 \mathrm{mg}$, $\left.[\alpha]_{D}^{24}+108^{\circ}\left(c 1, \mathrm{H}_{2} \mathrm{O}\right), \mathrm{m} / \mathrm{e} 274 \mathrm{M}^{+}\right)$as shown in Fig. 1.

Comparison of the ${ }^{12} \mathrm{C}$ NMR spectra data of
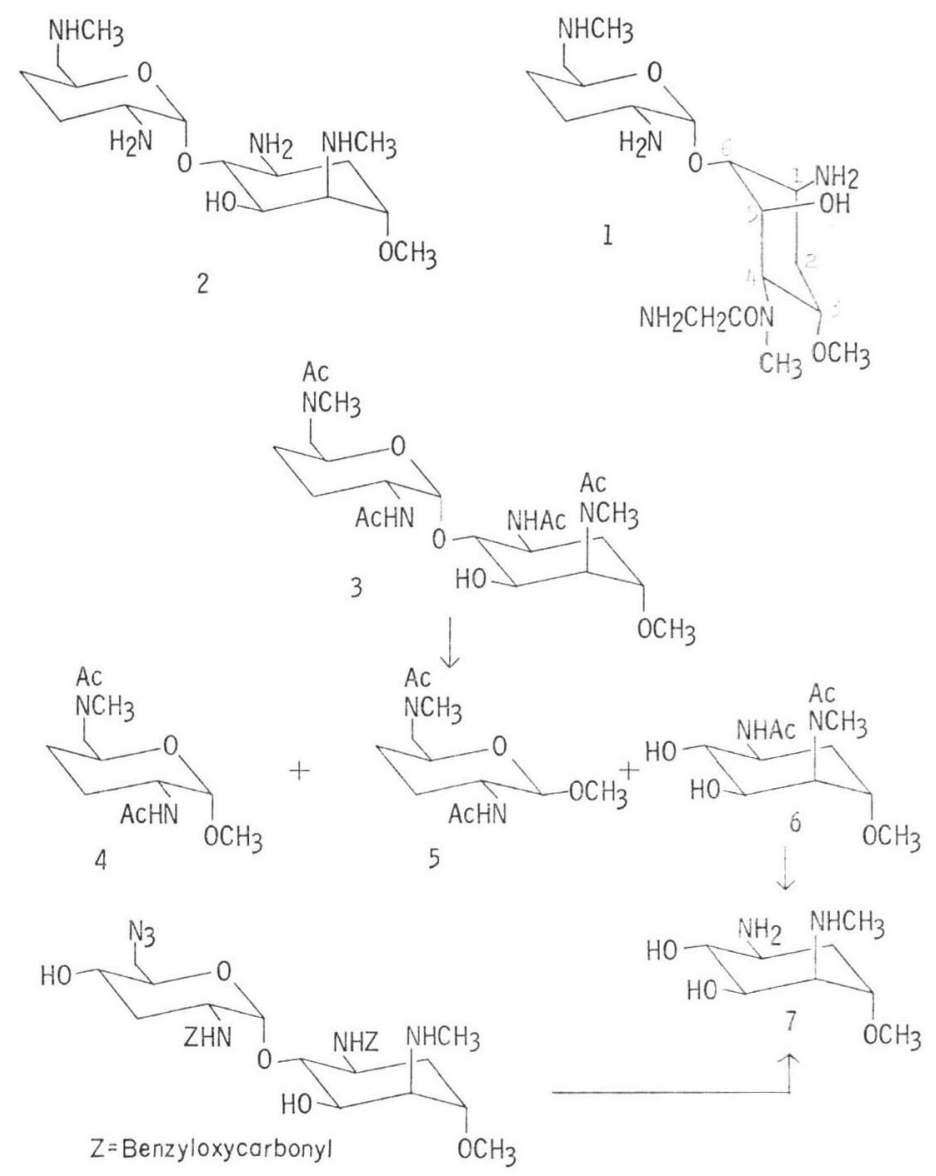
$\mathbf{1}$ and $\mathbf{2}$ with those of purpurosamine-containing antibiotics suggests the sugar moieties of sannamycins $\mathrm{A}$ and $\mathrm{B}$ to be 6-N-methylpurpurosamine $\mathrm{C}$, known as a component of sagamicin ${ }^{4}$ (gentamicin $\mathrm{C}_{2 \mathrm{~b}}$ ). Then we synthesized methyl 2,6-di-N-acetyl-6-N-methylpurpurosamine C $\left([\alpha]_{\mathrm{D}}^{23}+135^{\circ}\left(c \quad 1, \mathrm{H}_{2} \mathrm{O}\right)\right)$ from methyl N-benzyloxycarbonyl- $\alpha$-D-glucosaminide using the TIPSON CoHen method ${ }^{5)}$ as shown in Scheme 1 in an overall yield $36 \%$. This synthetic compound was found to be identical with $\mathbf{4}$ obtained from sannamycin B in specific rotation, IR and ${ }^{1} \mathrm{H}$ NMR spectra.

Treatment of $6(200 \mathrm{mg}, 0.78 \mathrm{mmol})$ with $4 \mathrm{~N}$ sodium hydroxide $(12 \mathrm{ml})$ at $110^{\circ} \mathrm{C}$ for 5 hours afforded the aminocyclitol $(7,117 \mathrm{mg}),[\alpha]_{D}^{24}-44^{\circ}$ (c $1, \mathrm{H}_{2} \mathrm{O}$ ), m/e $190 \mathrm{M}^{+}$, Anal. $\mathrm{C}_{8} \mathrm{H}_{18} \mathrm{~N}_{2} \mathrm{O}_{3} \cdot \mathrm{H}_{2} \mathrm{O}$. As shown in Tables 1, 2 and 3, the structure of 7 was shown to be 2-deoxyfortamine or its mirror image by the ${ }^{1} \mathrm{H}$ and ${ }^{13} \mathrm{C}$ NMR spectra of 7 .

In order to prove the structure and absolute configuration of 7, 2-deoxyfortamine was afforded by the methanolysis of 6-O-(6-azido-2-N-benzyloxycarbonyl-6-deoxy - $\alpha$-lividosaminyl) - 1 - Nbenzyloxycarbonyl-2-deoxyfortamine ${ }^{6)}(8)$ prepar-

Table 1. Chemical shifts of ${ }^{1} \mathrm{H}$ NMR spectra.

\begin{tabular}{|c|c|c|c|}
\hline \multirow[b]{2}{*}{ Proton } & \multicolumn{3}{|c|}{ Chemical shifts (ppm) } \\
\hline & $\begin{array}{l}\text { Sanna- } \\
\text { mycin A } \\
\text { (1) }\end{array}$ & $\begin{array}{l}\text { Sanna- } \\
\text { mycin B } \\
(2)\end{array}$ & $\begin{array}{l}\text { Amino- } \\
\text { cyclitol } \\
\text { (7) }\end{array}$ \\
\hline $1^{\prime}$ & 5.37 & 5.54 & \\
\hline $2^{\prime}$ & 3.28 & $\sim 3.4$ & \\
\hline $\left.\begin{array}{l}3^{\prime} \\
4^{\prime}\end{array}\right\}$ & $1.8 \sim 2.4$ & $1.8 \sim 2.4$ & \\
\hline $5^{\prime}$ & $\sim 3.95$ & $\sim 4.5$ & \\
\hline $6^{\prime}$ & 3.10 & 3.10 & \\
\hline 1 & $\sim 3.95$ & 3.51 & 3.27 \\
\hline $2 a x$. & 2.39 & $\sim 2.1$ & 2.05 \\
\hline 2eq. & 2.90 & 2.55 & 2.54 \\
\hline 3 & 4.68 & 4.23 & 4.23 \\
\hline 4 & 5.10 & $\sim 3.4$ & 3.48 \\
\hline 5 & 4.65 & 4.51 & 4.26 \\
\hline 6 & 4.31 & 4.03 & 3.83 \\
\hline $6^{\prime} \mathrm{N}-\mathrm{CH}_{3}$ & 2.81 & 2.81 & \\
\hline $4 \mathrm{~N}-\mathrm{CH}_{3}$ & 3.58 & 2.85 & 2.83 \\
\hline $\mathrm{O}-\mathrm{CH}_{3}$ & 3.90 & 3.91 & 3.87 \\
\hline Gly. $\mathrm{CH}_{2}$ & 4.04 & & \\
\hline
\end{tabular}

Chemical shifts of ${ }^{1} \mathrm{H}$ NMR spectra were measured in $\mathrm{D}_{2} \mathrm{O}$ using TMS as the external reference. ed starting from lividamine. They exhibited identical mobilities on thin-layer chromatograms virtually, superimposable IR and NMR spectra and similar optical rotations.

The position of linkage of the aminosugar to the aminocyclitol was decided by the ${ }^{13} \mathrm{C}$ NMR spectra of 2 and 7 (Table 3). The significant change in assignment for $\mathrm{C}_{6}$ in $2(85.3 \mathrm{ppm})$ and

Table 2. Coupling constants of ${ }^{1} \mathrm{H}$ NMR spectra.

\begin{tabular}{|c|c|c|c|}
\hline \multirow[b]{2}{*}{ Coupling } & \multicolumn{3}{|c|}{ Coupling constants $(\mathrm{Hz})$} \\
\hline & $\begin{array}{l}\text { Sanna- } \\
\text { mycin A } \\
\text { (1) }\end{array}$ & $\begin{array}{l}\text { Sanna- } \\
\text { mycin B } \\
(2)\end{array}$ & $\begin{array}{l}\text { Amino- } \\
\text { cyclitol } \\
\text { (7) }\end{array}$ \\
\hline $\mathbf{J}_{1^{\prime}, 2^{\prime}}$ & 3.5 & 3.7 & \\
\hline $\mathbf{J}_{5^{\prime}, 6^{\prime}}$ & 6.0 & 5.5 & \\
\hline $\mathbf{J}_{1,2 a x}$ & 4.0 & 10.0 & 12.0 \\
\hline $\mathbf{J}_{1,2 \text { eq. }}$ & 3.0 & 4.2 & 4.5 \\
\hline $\mathbf{J}_{2 \mathrm{eq}, 2 \mathrm{ax} .}$ & 13.5 & 14.5 & 14.0 \\
\hline $\mathbf{J}_{2 \mathrm{ax}, 3}$ & 11.0 & 4.0 & 3.3 \\
\hline $\mathbf{J}_{2 \mathrm{eq},, 3}$ & 4.2 & 4.5 & 4.0 \\
\hline $\mathbf{J}_{3,4}$ & 11.5 & 4.0 & 3.3 \\
\hline $\mathbf{J}_{4,5}$ & 3.0 & 4.2 & 4.5 \\
\hline $\mathbf{J}_{5,6}$ & 3.0 & 8.5 & 9.5 \\
\hline $\mathbf{J}_{1,6}$ & 3.0 & 8.5 & 9.5 \\
\hline
\end{tabular}

Table 3. Chemical shifts of ${ }^{13} \mathrm{C}$ NMR spectra.

\begin{tabular}{l|c|c}
\hline \multirow{2}{*}{ Carbon } & \multicolumn{2}{|c}{ Chemical shifts (ppm) } \\
\cline { 2 - 3 } & $\begin{array}{c}\text { Sannamycin B } \\
\mathbf{( 2 )}\end{array}$ & $\begin{array}{c}\text { Aminocyclitol } \\
(\mathbf{7})\end{array}$ \\
\hline $1^{\prime}$ & 102.4 & \\
$2^{\prime}$ & 50.9 & \\
$3^{\prime}$ & 27.5 & \\
$4^{\prime}$ & 29.3 & \\
$5^{\prime}$ & 69.5 & \\
$6^{\prime}$ & 56.1 & \\
1 & 49.6 & 50.4 \\
2 & 31.6 & 31.3 \\
3 & 76.7 & 77.0 \\
4 & 63.7 & 63.8 \\
5 & 71.7 & 72.2 \\
6 & 85.3 & 76.4 \\
$6^{\prime}-\mathrm{N}^{\prime}-\mathrm{CH}_{3}$ & 36.2 & \\
$4-\mathrm{N}-\mathrm{CH}_{3}$ & 35.3 & 35.8 \\
O- $\mathrm{CH}_{3}$ & 57.3 & 57.3 \\
\hline
\end{tabular}

The ${ }^{13} \mathrm{C}$-FT NMR spectra were taken with a JNM-FX-100 spectrometer.

Samples were dissolved in $\mathrm{D}_{2} \mathrm{O}$ containing dioxane as the internal reference $(67.4 \mathrm{ppm})$. 
Scheme 1 .

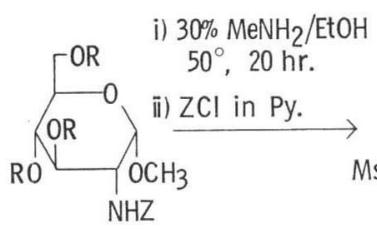

$A: R=H$

$B: R=M s$

$(a)_{D}^{22}(\mathrm{c} 1) \quad \mathrm{B}:+82^{\circ}\left(\mathrm{CHCl}_{3}\right)$

$+67^{\circ}\left(\mathrm{CHCl}_{3}\right)$

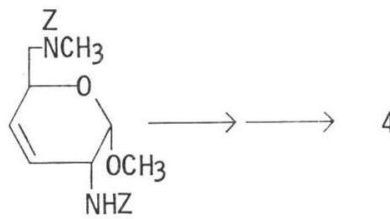

$-26^{\circ}\left(\mathrm{CHCl}_{3}\right)$

$+135^{\circ}\left(\mathrm{H}_{2} \mathrm{O}\right)$

Higashimurayama, Tokyo 189, Japan

(Received August 15, 1979)

\section{References}

1) Deushi, T.; A. Iwasaki, K. Kamiya, T. MizoGUCHI, M. NAKayama, H. ITOH \& T. MORI: New aminoglycoside antibiotics, sannamycin. J. Antibiotics 32: 1061 1065, 1979

2) Deushi, T.; M. Nakayama, I. Watanabe, T. Mori, H. Naganawa \& H. Umezawa: A new broad-spectrum aminoglycoside antibiotic complex, sporaricin. III. The structures of sporaricins A and B. J. Antibiotics 32:187 192, 1979

3) Egan, R. S.; R. S. Stanaszek, M. Cirovic, S. L. Mueller, J. Tadanier, J. R. Martin, P. Collum, A. W. Goldstein, R. L. DeVault, A. C. Sinclair, E. E. Fager \& L. A. Mitscher: Fortimicins $\mathrm{A}$ and $\mathrm{B}$, new aminoglycoside antibiotics. III. Structural identification. J. Antibiotics 30: 552 563, 1977

4) Egan, R. S.; R. L. DeVault, S. L. Mueller, M. I. Levenberg, A. C. Sinclair \& R. S. StanAszeK: A new antibiotic XK-62-2. III. The structure of XK-62-2, a new gentamicin C complex antibiotic. J. Antibiotics 28: 29 34, 1975

5) Umezawa, S.; Y. OKazaKi \& T. Tsuchiya: Studies on aminosugars. XXI. Synthesis of 3,4dideoxy-3-enosides and the corresponding 3,4dideoxysugars. Bull. Chem. Soc. Jap. 45: 3619 3624, 1972

6) Yamaguchi, T.; Y. Kyotani, I. Watanabe, S. Sato, Y. Takahashi, M. Nagakura \& T. MORI: Syntheses of sporaricin analogues. 2-deoxy-4-N-glycyl-6-O-( $\alpha$-nebrosaminyl) fortamine and its 3-de-O-methyl compound. J. Antibiotics 32 (11): 1979 (in press)

Tokyo Research Laboratories, Kowa Co., Ltd., 